\title{
EDITORIAL
}

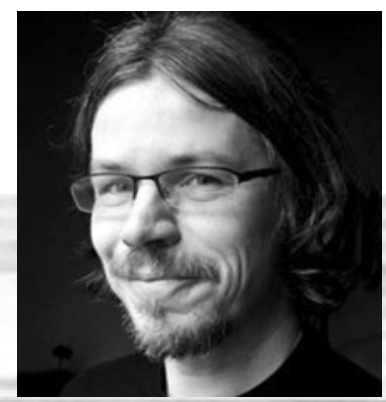

Peter Pagel

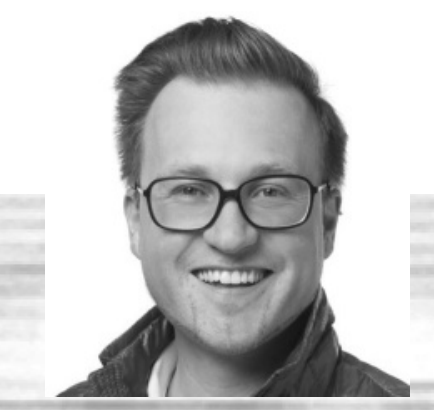

Edy Portmann

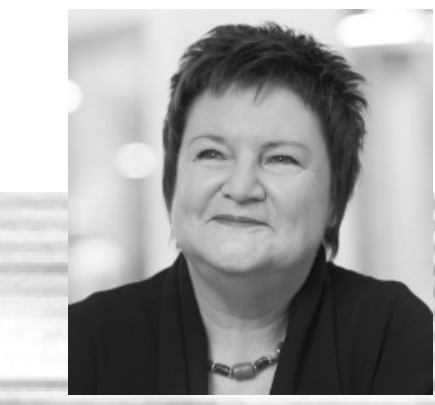

Karin Vey

\section{Cognitive Computing}

Es gibt die Hoffnung, dass die rasante Weiterentwicklung der Künstlichen Intelligenz (KI) das Zusammenspiel zwischen Mensch und Computer auf ein neues Niveau heben könnte. Computer sollen lernen, Menschen zu verstehen, zum Beispiel zu erkennen, wie sich ihr Gegenüber gerade fühlt. Dieses sogenannte Cognitive Computing wird in der Science-Fiction schon lange thematisiert, jetzt kommt es Schritt für Schritt in der Wirklichkeit an.

Der Chefredakteur des Informatik Spektrums, Peter Pagel, dazu im Gespräch mit den beiden Gastherausgebern dieses Themenheftes, Edy Portmann und Karin Vey.

Peter Pagel: Oft ist die Rede von intelligenten Maschinen. Assoziiert wird dabei häufig, dass die KI menschliches Denken simuliert. Ist das der Fall oder worin unterscheidet sich die Intelligenz von Computern von der des Menschen?

Edy Portmann: Ich möchte zuallererst einmal festhalten, dass es heute keine eindeutige Definition von Intelligenz gibt. Was ist Intelligenz? Ist eine Ameise intelligent? Ist es ein selbstfahrendes Auto? Darauf gibt es so viele Antworten, wie es Experten gibt. Die Idee hingegen, menschliches Denken zu simulieren, ist schon ziemlich alt. Beispielsweise charakterisierte der französische Arzt und Philosoph Julien Offray de La Mettrie schon im Jahre 1748 in seinem Werk "L'homme machine" den Menschen bereits als Maschine. Seit es mechanische - oder später elektronische - Systeme gibt, versucht der Mensch, Maschinen zu konstruieren, die sprechen, zuhören, intelligent interagieren und antworten können.

Dabei möchten die Menschen gerne so denken, wie Computer funktionieren, um mit diesen besser zu kommunizieren. Eine andere Idee könnte jedoch auch sein, Maschinen bauen zu wollen, die so arbeiten, dass ihre Outputs dem menschlichen Output ihres Denkens ähneln. Diese Idee beschrieb der amerikanische KI-Forscher Lotfi A. Zadeh beispielsweise schon 1984 in einem Artikel „Making Computers think like People." Die Weiterführung dieser Idee führt uns zu kollektiver Intelligenz, welche Mensch und Maschine verbindet, sodass sich diese gemeinsam intelligenter verhalten, als es ein Mensch oder ein Computer alleine tun könnte. Diese Art der Intelligenz, welche eine Brücke zwischen menschlicher und künstlicher Intelligenz schlägt, unterstützt uns schon heute, immer erfolgreicher zu werden.

Peter Pagel: Wie berechtigt ist die Sorge, dass Künstliche Intelligenz mittelfristig zu gewaltigen Verlusten an Arbeitsplätzen führen wird?

Karin Vey: Wir befinden uns inmitten eines tiefgreifenden Transformationsprozesses. Kognitive Assistenten werden uns helfen, aus Big Data Sinn zu machen und in Zusammenarbeit mit dem Menschen evidenzbasierte, innovative und personalisierte Lösungen zu ermöglichen. Es geht um die Erweiterung und Ergänzung der menschlichen Intelligenz. Im Bereich der Wissensarbeit werden zum Beispiel repetitive Aufgaben, Recherchen, Spreadsheet-Management zunehmend von den Maschinen übernommen. Menschen werden sich vermehrt höherwertigen Aufgaben zuwenden - etwa die richtigen Fragen stellen, Ziele und Visionen kreieren oder Mitarbeiter coachen.

Die künftige Arbeitswelt bietet für den Menschen eine Vielzahl an Tätigkeitsfeldern. Da sind natürlich jene Experten zu nennen, die mit den Systemen arbeiten oder Technologie entwickeln. Allein im Bereich Cyber-Security fehlen in den nächsten Jahren weltweit Hunderttausende an Mitarbeitern. Es sind jedoch keineswegs nur technische Skills gefragt. Viele Berufe werden sich etwa dergestalt verändern, dass in einer komplementären Partnerschaft zwischen 


\section{$\{$ EDITORIAL}

Mensch und Maschine menschliche Kernkompetenzen Trumpf sein werden. Es gilt, unsere ureigenen menschlichen Fähigkeiten wieder mehr in den Fokus zu rücken und kritisches Denken, Intuition oder Kreativität in besonderer Weise zu kultivieren.

Ein gutes Beispiel hierfür ist der Beruf des Arztes. Kognitive Systeme bereiten elektronische Krankenakte, Testergebnisse, Beschreibungen von Symptomen und wissenschaftliche Erkenntnisse für den Arzt auf, liefern Analysen und Empfehlungen für Diagnose und Therapie. Damit wird die Basis für personalisierte Medizin geschaffen. Nur der Arzt aber hat eine holistische Wahrnehmung der Situation, kann auf feinste Reaktionen seines Gegenübers reagieren und seine Intuition sprechen lassen. In besonderer Weise zum Heilungserfolg dürfte dazu beitragen, dass Ärzte sich wieder mehr dem Patienten zuwenden und etwa mit ihm darüber sprechen können, was eine bestimmte Diagnose denn für diesen Menschen bedeutet. Menschliche Empathie, die bekanntermaßen als essentieller Faktor zum Heilungsfaktor beiträgt, kann wieder voll zum Tragen kommen.

In bestimmten Berufsfeldern wird die Nachfrage nach menschlichen Kernkompetenzen sogar stark steigen, etwa in der Pflege oder im Bereich Gesundheit und Wellness. Als Berufe mit besten Aussichten gelten auch Handwerksberufe. Dazu kommt dann z. B. noch die Wiederbelebung von schon fast ausgestorbenen Berufen oder Tätigkeiten, die wir aus ethischen oder rechtlichen Gründen nicht durch eine Maschine ausführen lassen wollen. Last but not least wären dann noch innovative Tätigkeiten im Dienstleistungssektor zu nennen, die durch menschliche Kreativität, Digitalisierung und weltweite Vernetzung erst möglich werden. Der Raum der Möglichkeiten für den Menschen scheint sich eher noch zu erweitern.

Edy Portmann: Es geht also nicht um die Ablösung der Menschen, sondern vielmehr um eine Symbiose zwischen menschlicher und künstlicher Intelligenz. Wir beschäftigen uns heute damit, biologische, menschliche und künstliche Intelligenz miteinander zu verbinden, um gemeinsam erfolgreicher zu werden. Der Mensch wird nicht einfach wegrationalisiert, sondern er erhält durch diese Art der kollektiven Intelligenz die Möglichkeit, sich weiterzuentwickeln.

Der Philosoph Michel Serres glaubt, dass der Mensch jedes Mal einen Schritt über sich selbst hinauswuchs, wenn er sich eines umweltbedingten Zustandes entledigen konnte. Nachdem er den aufrechten Gang erlernt hatte, konnte er die nun freien Hände nutzen, um Werkzeuge zu erschaffen. Die Erfindung der Schrift befreite ihn von der mündlichen Überlieferung und ermöglichte es ihm, Wissen zu sammeln und zu verstetigen. Ähnlich verhält es sich schon heute und in Zukunft wohl noch mehr, mit künstlicher Intelligenz, welche den Menschen von Routineaufgaben und Alltagsverrichtungen entlasten kann. Er kann sich stattdessen auf kreative und schöpferische Tätigkeiten konzentrieren.

Peter Pagel: Können Sie kurz die Entwicklung des Cognitive Computing von den Anfängen bis heute skizzieren?

Karin Vey: Cognitive Computing ist eine recht neue Entwicklung im Bereich der KI-Forschung. Als das IBM WatsonSystem 2011 in der amerikanischen Quizshow "Jeopardy!" gegen die zwei weltbesten menschlichen Spieler gewann, beendete es durch seine Performance den letzten KI-Winter und läutete die Ära des Cognitive Computings ein. Der Auftritt in Jeopardy war einmalig und ein Benchmark. Unmittelbar danach hat das System seine Arbeit in verschiedenen Industrien aufgenommen, allen voran dem Gesundheitswesen. Heute gibt es auch in vielen anderen Bereichen wie dem Finanz- und Versicherungssektor, Bildungsorganisationen oder der Polizei spezifische Watson-Lösungen, die bei der Entscheidungsfindung unterstützen.

2011 war das System noch ein riesiger Supercomputer. Vor einigen Jahren haben wir es in seine kognitiven Funktionalitäten „zerlegt" und diese als APIs auf der Cloud-Plattform von IBM zur Verfügung gestellt. Diese Öffnung war ein wichtiger Schritt hin zu niederschwelligen Lösungen und dem Aufbau eines inspirierenden Ökosystems. Heute können Sie sich als Entwickler eine kognitive Lösung selbst bauen und einfach mit ihrer Kreditkarte zahlen.

Edy Portmann: Heute eruiere ich zudem eine weitere, erfolgversprechende Form von Cognitive Computing, welche meiner Meinung nach bisher noch zu wenig beachtet wurde, im Soft Computing. Damit Maschinen irgendwann einmal wie Menschen „denken“ können, müssen ihre Fähigkeiten mit „Zahlen" zu rechnen, durch solche mit „Worten und Wahrnehmungen" zu rechnen, erweitert werden. Die zugrundeliegende interdisziplinäre Herangehensweise, welche ab 1990 auch humanistische Ansätze in die KI-Forschung brachte, ermöglicht vielleicht einmal das menschliche Denken, welches eher annähernd als exakt ist, synthetisch nachzuahmen. Gemäß Lotfi A. Zadeh, dem Vater des Soft 
Computings, bergen die in der traditionellen KI verankerten Vorstellungen von Präzision, Sicherheit und Genauigkeit nämlich große Kosten, welche durch Methoden wie Fuzzy-Logik, künstliche neuronale Netze, evolutionäres Rechnen, etc. umgangen werden können.

Solch heranreifende Ansätze, welche die Bildung von kollektiver Intelligenz begünstigen, zielen darauf ab, die vermeintliche Dualität zwischen Natur und Technik zu widerlegen. Sie basieren auf der Vorstellung, dass natürliche und künstliche Intelligenz Entwicklungsstufen voneinander darstellen, die wie Zahnräder ineinandergreifen, ohne einander obsolet zu machen. In diesem Sinne können Dinge, Werkzeuge, Technik und Technologie als Teile von Menschen verstanden werden, die unsere Intelligenz, unser Denken und unsere Wahrnehmung über ihre Grenzen hinaus erweitern. Diese Art des Computings hilft uns wohl dereinst, Probleme ganzheitlicher zu lösen. Wir brauchen es, um neue Möglichkeiten zu schaffen und unser Bewusstsein auszubauen, um unsere Reichweiten und, letztendlich, unseren Willen zu erweitern. Soft Computing bedient sich dazu der Metapher des Werkzeugkastens, aus welchem jeweils das Werkzeug entnommen wird, welches der Lösung eines Problems dient.

Peter Pagel: Und was erwarten Sie für die Zukunft?

Edy Portmann: Unser als menschliches Kollektiv angestrebtes Ziel liegt wohl darin, das Konzept der Intelligenz zu entschlüsseln. Jedoch werden wir nie „echte“ künstliche Intelligenz schaffen können, solange wir die unterliegende menschliche Intelligenz nicht verstehen. Die Natur entwickelte diese als Hilfsmittel, um die Menschen erfolgreich durch eine sich stetig ändernde Umwelt zu führen. Daraus entstanden dann beispielsweise unser Verhalten, unsere Emotionen sowie unser Bewusstsein. Diese Faktoren müssen bei der Entwicklung von echter künstlicher Intelligenz mitberücksichtigt werden. Für die Zukunft müssen wir also unbedingt über die Limitationen heutiger KI hinwegkommen. Ein Beispiel einer Kl-Limitation ist etwa die große Datenmenge, welche für maschinelles Lernen heute - noch notwendig ist, aber beim menschlichen Lernen eine untergeordnete Rolle zu spielen scheint. Vielleicht sollten wir uns also auf solche Beispiele konzentrieren, um die unterliegenden Prinzipien der menschlichen Intelligenz zu verstehen.

Karin Vey: Künstliche Intelligenz wird in den nächsten Jahren noch weiter an Bedeutung gewinnen und zur wichtigsten fundamentalen Technologie werden. Schon heute sehen wir, dass zahlreiche Unternehmen eine eigene Kl-Abteilung einrichten. So ähnlich verhielt es sich vor einigen Jahrzehnten mit der IT - die sich heute überhaupt nicht mehr wegdenken lässt und eine essentielle Grundlage für unsere Ökonomie ist. KI wird in nächster Zeit in Hinblick auf Geschwindigkeit und Effizienz noch deutlich leistungsstärker und ähnlich unentbehrlich werden, wie es die IT schon heute ist. Für bestimmte Anwendungsbereiche wie Kryptographie und Pharma wird die Entwicklung des Quantum Computings der Schlüssel sein für Leistungen der künstlichen Intelligenz, die die Grenzen des Möglichkeitsraums klassischer von Neumann-Computer signifikant überschreitet. Zentral ist, dass die KI die menschliche Intelligenz zunehmend augmentierend unterstützen wird. Der Blick aufs "große Ganze”, Bewusstsein und Selbstreflexion bleibt wohl aber auch in Zukunft dem Menschen vorbehalten.

Peter Pagel: Bleibt Kreativität eine Domäne des Menschen oder gibt es so etwas wie kreative Computer?

Karin Vey: Zunächst ist wichtig festzustellen, dass wir nicht immer vom selben Sachverhalt sprechen, wenn wir den Begriff der Kreativität verwenden. Eine kreative Handlung kann, je nach Standpunkt, vieles bedeuten und in Ausmaß und Komplexität sehr verschieden sein: Sowohl die bloße Rekombination vorhandener Fakten, als auch die Schaffung eines künstlerischen Meisterwerks, das ein ganzes Genre transformieren oder gar neu begründen kann, wird als "kreativ" bezeichnet. Während kognitive Systeme eine rekombinatorische Kreativität im zunehmenden Masse gut beherrschen, wird die Fähigkeit zur transformativen Kreativität wohl auch in Zukunft dem Menschen vorbehalten bleiben. Bereiche wie Körperlichkeit, Individualität und Subjektivität, Bewusstsein, das Unbewusste und Sinnlichkeit scheinen hierfür notwendig, jedoch von Systemen nicht oder nur zum Teil umsetzbar zu sein.

Selbst wenn jedoch die Frage der technischen Machbarkeit gelöst wäre, bliebe es äußerst fraglich, ob Maschinen überhaupt zur transformativen Kreativität befähigt werden sollten. Kreativität verlangt immer auch die Ablösung des Bestehenden. Es ist der Kern jeder kreativen Handlung, das Alte zu zerstören und durch Neues zu ersetzen. Die Freiheit, dies zu denken und zu postulieren, sollte auch in Zukunft nur dem Menschen zugestanden und im gesellschaftlichen Diskurs fortentwickelt werden. Maschinen jedoch, können uns Impulse geben, unsere Kreativität weiter zu entwickeln. 


\section{$\{$ EDITORIAL}

Peter Pagel: Werden wir durch den Einsatz künstlicher Intelligenz in die Lage versetzt, die großen Herausforderungen der Menschheit, wie etwa den Klimawandel zu meistern?

Edy Portmann: Gerade Herausforderungen wie der Klimawandel, bei welchem wir für den Fortbestand der Menschheit wohl zwingend auf intelligente Lösungen angewiesen sind, können von künstlicher Intelligenz stark profitieren. Zurzeit forschen die daran interessierten Disziplinen aber noch relativ isoliert und sind höchstens interdisziplinär organisiert. Eine intelligente Lösung wird jedoch nur dann zustande kommen, wenn die Forscher über ihre Grenzen hinausgehen und sie sich dadurch - unter Einbezug der Gesellschaft, Politik und Wirtschaft - gemeinsam anti- oder transdisziplinär an die Herausforderungen macht.

Die Verschmelzung von menschlicher und künstlicher Intelligenz, welche zur bereits genannten erweiterte kollektiven Intelligenz von Menschen und Maschinen führt, kann uns auch hier unterstützen. Denn die Forscher der verschiedenen Disziplinen verbinden sich nun nicht mehr nur untereinander, sondern suchen auch Kontakt zu relevanten und interessierten Stakeholdern. Dabei werden sie wohl immer mehr von Algorithmen unterstützt, welche auf maschinellem Lernen beruhend, die richtigen Stakeholder zusammenzuführen vermögen. Dieses, auf kollektiver Intelligenz gestützte, gemeinsame Forschen erhöhen - hoffentlich - die Chance für eine Meisterung des Klimawandels genügend, um unseren Fortbestand zu garantieren.

Peter Pagel: Herr Portmann, Frau Vey, besten Dank für das Gespräch. Es bleibt also spannend.

Zum Schluss dieses editorischen Interviews möchte ich mich gern noch kurz bei Ihnen vorstellen. Ich bin seit diesem Jahr Chefredakteur des Informatik Spektrums. Im vergangenen Jahr habe ich mir diese Verantwortung noch mit dem langjährigen Chefredakteur Hermann Engesser geteilt, jetzt geht diese Zeit des Übergangs dem Ende entgegen. Ich bin seit gut neun Jahren Chefredakteur von Wirtschaftsinformatik \& Management, ein Magazin, das einige von Ihnen sicherlich gut kennen. Auch zuvor hatte ich in meinem beruflichen und privaten Leben immer wieder mit Informationstechnologie zu tun. Spätestens mein erster Besuch auf der Hannover-Messe 1984 - ich durfte meinen Vater begleiten - weckte in mir die Faszination für diese Branche. Der dort gezeigte erste Apple Macintosh - mit Maus und Icons - ließ bereits ahnen, wie Desktop-Computer bald aussehen würden. Besonders angetan haben es mir immer Computergrafik, Nutzerfreundlichkeit und Künstliche Intelligenz. Begeistern kann ich mich aber für nahezu jeden Bereich der Informatik. Die enorme Vielfalt dieses Gebiets und die große Innovationskraft sorgen dafür, dass die Beschäftigung damit nie langweilig wird.

\section{Herzliche Grüße \\ Peter Pagel \\ Chefredakteur}

Zum Schluss noch eine wichtige Mitteilung in eigener Sache. Frank Puppe und Brigitte Bartsch-Spörl geben ab diesem Jahr die Betreuung der Rubrik "Aktuelles Schlagwort" ab. Ihr Nachfolger wird Thomas Ludwig sein. Wir danken Frank Puppe und Brigitte Bartsch-Spörl für viele Jahre hervorragender Zusammenarbeit und freuen uns auf eine ebensolche mit Thomas Ludwig.

\section{Arndt Bode und Peter Pagel}

